\title{
Using Public Health to Prevent Air-Borne Pandemics Rather than Medical Science Responding to Air-Borne Pandemics
}

\author{
Bahman Zohuri ${ }^{1,2,3 *}$ \\ ${ }^{1}$ Adjunct Professor, Business Analytics School, Golden Gate University, Ageno School of \\ Business, San Francisco, California, USA \\ ${ }^{2}$ Galaxy Advanced Engineering, Albuquerque, New Mexico, CEO, USA \\ ${ }^{3}$ Adjunct Professor, Computer Science and Electrical, Computer Engineering, Interna- \\ tional Technology University, Santa Clara, California, USA \\ *Corresponding Author: Bahman Zohuri, Adjunct Professor, Artificial Intelligence \\ and Machine Learning, Business Analytics School, Golden Gate University, Ageno \\ School of Business, San Francisco, California, USA.
}

Received: Bahman Zohuri

Published: June 23, 2021

(C) All rights are reserved by Bahman Zohuri.

\begin{abstract}
The fight against the pandemic is not going well-but not for the reasons many people with political ideologies believe. With President Biden Whitehouse in place, he is facing uphill battle July 4 vaccination goal of $70 \%$ of adults with at least one dose of COVID-19 vaccine arm injection, according to recent national news CNN analysis. But the administration continuous to push ahead with new and initiatives, and key players remain hopeful that the goal will be met. With Johnson and Johnson vaccine as a one-shot dose being out of market for any medical reason, people need two shots of dose against COVID-19 virous. But, with new different variety of this pandemics coming out of United Kingdom and as recent one reported coming out of Vietnam, are we really protected, even if we complete the goal of $70 \%$ by $4^{\text {th }}$ of July as Biden administration is targeting. Currently, $63 \%$ of adults in the US have received one dose of Covid-19 vaccine, and an average of about 371,000 adults were added to that total each day last week, according to data from the US Centers for Disease Control (CDC) and Prevention. But closer to 564,000 adults would need to initiate vaccination each day to reach Biden's goal by July 4 -- a rate that the US has only consistently fallen behind in the past week or so. The holiday weekend might have delayed some data reporting, but daily vaccinations had dipped below the required pace a few times before this week. Another 18 million adults will need to receive at least one dose to reach $70 \%$. At the current pace of vaccination, the US would hit about $68 \%$ of adults on July 4 -- falling short by about 6 million adults. The full 70\% would be reached about two weeks later. Even so, 12 states have already met Biden's goal: California, Connecticut, Hawaii, Massachusetts, Maryland, Maine, New Hampshire, New Jersey, New Mexico, Pennsylvania, Rhode Island, and Vermont. Eight others are on track to reach $70 \%$ of adults by July 4: Colorado, Delaware, Illinois, Minnesota, New York, Oregon, Virginia, and Washington, as well as Washington, DC. But three states -- Alabama, Louisiana, and Mississippi -- might not even vaccinate half of their adult residents with at least one dose by July 4 at their current pace. In this article we take a look at way where public health would enhance prevention of air born pandemics rather than just medical science such as full-vaccination target of entire population nation-wide and globally as well.
\end{abstract}

Keywords: Pandemic; COVID-19; Vaccine and Vaccination Artificial Intelligence; Machine Learning; Deep Learning; Drug Delivery and Administration; Drug Distribution

\section{Introduction}

By definition, a pandemic occurs when each infected person on the average infects more than one other person. It stops when each infected person infects less than one person. The end of a pandemic does not imply that the disease disappears-only that there are small local outbreaks and no large outbreaks. One starts with three observations about this pandemic. Recent worldwide COVID-19 pandemic, for example, has put tremendous pressure on pharmaceuticals scientists to be in quest of new drugs and vaccine to treat such deadly disease, and at this point, nobody knows what price tag 
has been estimated to develop such a vaccine by multi-drug companies involved with research and development of the drug.

The pandemic that has killed over a half-million Americans is an unintentional failure of the medical science community and a consequence of their capture of the public health establishment. The pandemic outcome was predictable. For decades the medical science community has been selling vaccines as the first line of defense against the air-borne flu that kills about 40,000 Americans in an average year. Sooner or later another air-borne virus would find home in the environmental niche we created for the flu and take advantage of it. We were lucky with this pandemic. There have been pandemics that killed a quarter of the population-fortunately COVID-19 is not very deadly compared to many pandemics in the past.

The cause of this failure is obvious-viruses do not send emails a year in advance that they are coming to enable creating vaccines in advance. A pandemic response based on vaccines assures mass death. The situation is becoming worse. We have created the perfect environment for air-borne pandemics by building [1] large cities with high population densities [2], mass transit systems that create the perfect environment to transmit airborne diseases between people and [3] a global air transport industry. An air-borne virus that is highly infectious before people get sick and starts in a very large city will create a global pandemic before anybody knows of its existence. The federal government strategy using vaccines to stop air-borne pandemics assures failure today and into the futureto slow and too late.

\section{Public health strategy}

The central role of large cities is seen in the history of pandemics and in this pandemic. The first outbreak of this virus in the United States was in Washington State where it was contained because low-density housing and limited mass transit in Seattle that limited virus transmission rates. However, once the virus entered very large cities such as New York, the mass transit systems and high population densities made it unstoppable. This virus started in Wuhan, a city of 10 million people-and then got to New York City. If you want to stop air-borne global pandemics, either ban the existence of large western cities such as New York or adopt another pandemic control strategy.

There is an alternative public health strategy. We can stop this and other viruses by filtering air or killing the viruses in air with proper ventilation systems where there are large crowds. It is not necessary to clean all air-just air where many people are crowded together each day to get the disease transmission rate below one new infection for each person with the virus. Some industrial com- panies, some schools, some hotels, and my dentist have adopted this strategy.

This solution

- Works against all air-borne viruses,

- Does not require that the virus send an email in advance to provide time to develop a vaccine, and

- Does not depend upon convincing or forcing most people to be vaccinated or wear masks.

\section{Engineering solutions}

Engineering solutions are the historical solution to public health challenges. There are five ways to transmit diseases to people and in four of the ways we use engineering barriers as the first and primary defense: drinking (clean water and sewer), eating (cook food and packaging), sex (right partner and condoms), biting insects with malaria and other diseases (window screens and killing mosquitos) and breathing. The medical science community is the backup squad in four of these cases. Only with breathing do we use vaccines and the medical profession as the failed first line of defense.

Our dependence on vaccines against air-borne viruses is an accident of history. With the early success of some vaccines, the medical science community took over public health and implemented a major change in strategy from:

- Preventing disease transmission to people (sewer, clean water, food sterilization, kill mosquitos, etc.) to

- Vaccines so individuals do not get sick.

The body fights off the attack. Without that change in strategy, we would have installed filtered ventilation decades ago to stop transmission of air-borne diseases-just like we installed clean water against water-borne diseases.

The absurdity of the current approach is evident if one looks at industrial safety. The Occupational Safety and Health Administration requires clean air to protect workers against air-borne hazards from welding fumes in factories to lead particles where companies recycle batteries. If a lead battery plant used masks on all workers as a long-term substitute for clean air, it would be shut down. Lead, like Covid-19, is natural-it is the man-made indoor environment that makes it so dangerous. Yet OSHA is unwilling to require companies to provide a safe working environment for office workers and subway workers against air-borne viruses. It is a catastrophic worker-safety failure of the federal government. 
Engineering solutions are the historical way societies reduce most risks. In the 1800 s Chicago, Boston and many other cities burnt down because the cities were built of wood-the fire equivalent of a pandemic. Fire departments, the equivalent of the medical profession to diseases, were not able to stop these fires. The solutions were building codes that required using brick, concrete, stone and plaster in new buildings. The engineering fixes stopped city-wide fires. These solutions did not stop an occasional building from burning to the ground but no city-wide fires. When applied to a pandemic, the vaccines and hospitals become the backup squadlike the fire department.

Furthermore, almost all pandemics have been stopped by engineering. Water borne pandemics were stopped by sewer systems and clean water where we destroyed the environmental niche where these viruses grew and spread to man. For all we know, some new super virus has shown up in sewage or untreated water, but it has no effect on us because clean water and sewers stop all viruses. Malaria is controlled by draining the swamps and other methods to kill mosquitoes that transmit the disease to man.

We can stop this virus by filtering air or killing the viruses in air with proper ventilation systems where there are large crowds [4]. It is not necessary to clean all air-just where a lot of people are crowded together each day to get the disease transmission rate below one new infection for each person with the virus. The big industrial companies, some schools and my dentist have adopted this workable strategy. This solution works against all air-borne viruses and is compatible with western culture that values the individual with rule breaking. It is the equivalent of providing clean water rather than asking everyone to boil their water.

In many office, store, and factory environments this implies:

- Upgrading filters in the main ventilation systems and,

- Installing local cheap filter-fan systems that filter air to remove the viruses. Substantially higher air filtration rates are required to minimize transmission of the virus between people than used to heat and cool buildings. The low-cost option is local air-cleaning systems that consists of a high-quality filters with a fans-or potentially Ultra Violet (UV) sterilization units with fans. This pandemic would be partly under control if there had been a full push to put in clean air systems.
The fastest and simplest way to implement such changes is to pass legislation which makes the insurance industry liable for the cost consequences of the spread of COVID-19 in congested indoor spaces unless appropriate engineering changes are made to the buildings. Insurance is required to obtain loans on commercial property. Such legislation would bring to bear all the resources of banks and financial institutions that back commercial mortgages to immediately fix the problem.

Engineering solutions are the standard will-work option to reduce risk. In the 1800s Chicago, Boston and many other cities burnt down because the cities were built of wood-the fire equivalent of a pandemic. Fire departments, the equivalent of the medical profession to diseases, were not able to stop these fires. The solutions were building codes that required using brick, concrete, stone, and cement in new buildings. The engineering fix stopped city-wide fires. The engineering solutions did not stop an occasional building from burning to the ground but no city-wide fires. The same will be true if we use engineering to stop this air-borne pandemic.

\section{Engineering solutions costs, challenges and requirements}

The cost of clean filtered air is small wherever there is spacesuch as large grocery stores, classrooms and big-box retail stores. What is primarily needed are simple, cheap filter and fan systems. The cost in rural and suburban areas will be low. The most difficult ventilation challenges are in aircraft. Fortunately, commercial aircraft ventilation systems today have filters that remove virusesonly small changes in air distribution are needed to avoid mixing air between passengers. The cost to fix air distribution systems with new aircraft or when replace the interiors of old aircraft is close to zero.

The challenges today are in dense urban areas that enable global pandemics. The cost in new buildings is low-but retrofit will be expensive in buildings and mass transit where historically public health was either ignored or not a priority. The most expensive fixes may be existing subway cars-limited space to install safe filtered air systems without cutting off the top half of the car to provide space for equipment or using up significant passenger space. That is followed by energy-efficient green buildings that recycle air with low-grade filters to reduce energy costs (with sick building syndrome) and created an efficient way to transmit air-borne diseases between workers. The safest offices are the old offices that do not have central heat and air but radiators and windows. 
Last, there are politically-protected institutions such as Broadway where thousands of people sit tightly together for several hours exchanging air-borne diseases. Because people from across the country go to Broadway to see shows and then return home, it is a great way to quickly spread an air-borne pandemic across the country. The local high-school basketball game is far less dangerous because smaller crowds, any disease outbreak is local, and the building is filled only once a week-not multiple shows per day to jump-start air-borne pandemics.

The other requirement to stop air-borne pandemics is for sensors that enable every individual to know if they are in a location with high risk of spreading air-borne viruses from one person to another. We do not have detectors for particular viruses to tell us what places are dangerous; but, we have something that is far better. When you inhale air, you exhale carbon dioxide and viruses. The carbon dioxide content in the air around you goes up. We can install cheap carbon dioxide detectors in indoor public locations that read out carbon dioxide levels to nearby cell phones. The detectors tell you how much virus-laden air that you are breathing was in somebody else's lungs a few minutes ago. It is a warning system effective against all viruses. Some organizations today use such detectors to protect employees against Covid-when to open windows or have people go home early for safety. This is cheap and fast to install.

A requirement for carbon dioxide air monitors would have three impacts. Consumer boycotts would likely put out of business mass transit authorities and businesses that refuse to provide safe air. Second, any future business shutdowns to stop air-borne pandemics would be based on danger to the public-not political connections to the governor making decisions on which businesses are essential. Last, air monitors would provide a clear priority list of where to first fix old ventilation systems.

The biggest surprise of this airborne pandemic is that culture is the most important factor in determining outcomes. Confucian cultures (China, Japan, and South Korea) where the culture emphasizes the society over the individual were reasonably successful at stopping the virus. China was spectacularly successful because it combined a Confucian culture with a powerful state willing to use the full power of the state for force compliance. In contrast, the death rates of Europe and the United States are almost identical because western culture emphasizes the individual over the society. Differences in European versus American leadership, political systems and medical systems made only small differences in outcomes.

\section{Virous spread prevention}

In many western countries officials quickly imposed restrictions to limit disease transmission between people to stop the pandemic-but this was followed in a month or two with widespread disobedience that was often followed by riots. In each case the political leadership had to decide whether to loosen rules that slowed the spread the disease or imprison hundreds of thousands of people and shoot protesters as the riots spread. The result has been similar pandemic outcomes across democratic western states as politicians chose to reduce pandemic restrictions rather than face rebellion. In the U.S., the change in administrations did not result in major changes in outcomes or policies-renaming Project Warp Speed for vaccine production, requiring masks in federal facilities and a President wearing a face mask are minor incremental changes. President Biden chose to leave the governors making all the tough decisions. Western experts failed to account for culture and that failure resulted in bad advice and hundreds of thousands of added deaths. Their advice was better suited for the Chinese government than any western democracy.

The pandemic exposed an inherent weakness in western democratic cultures. Anybody who wishes to destroy the west now knows a workable strategy-find, create or evolve air-borne viruses that are infectious before sickness or death. That includes new mutations of Covid-19 that work around the vaccines-something that may be relatively easy to do.

The damage will be much higher in western than in many other cultures. It is a safe bet several countries and possibly some terrorist organizations are developing new air-borne viruses now that this weakness has been identified. From a national security perspective, there is no choice but to go to a public health strategy to prevent transmission of air-borne diseases between people (ventilation and sensors) than an after-the-fact year-late vaccine strategy.

\section{Conclusion}

In conclusion, as it was described in abstract and content of this article, dealing with such devasting and unknown disease, requires tremendous knowledge to be gathered in a short period of time, in order to have power of action and reaction how to deal with this global pandemic, both from granular and holistic point of view. 
To be powerful enough and being able to take on such horrible pandemic, we need to collect data globally to increase our information along with knowledge gathered from this information. Such process is way over beyond our own brain intelligence to process [5-7], thus we have no choice except to rely on Artificial Intelligence, Machine Learning and Deep Learning system tools to be augmented with the effort toward halting such disease on spot so we can prevent all its devasting adverse side-effects that we have recently encountered both Nationally and Internationally.

As we have seen as adverse side-effects of COVID-19, businesses are shutdown, economy has gone upside down, people have been out of their jobs and consequently suffering from lack of steady income to live their normal life, depression among the folks is on the rise due to being confined within their own home with stay at home order and we can go on and on with situation in hand with this pandemic.

Even though, some sort of countermeasure in form of new vaccinations are available to us to fight this horrible disease, we still have not managed to administer them to our arms at hundred percent delivery structure due to lack of an effective infrastructure either. On an all, we need to come up with better means of distribution and delivery system on top of research and development of such vaccine to halt this pandemic and this were we need Human Intelligence and Artificial Intelligence work as a team and handin-hand.

Finally, we can wrap our conclusion out by stating that, the founding fathers of this country believed that self-interest drove most decisions by men. They understood that self-interest creates scientific and moral blindness. Our pandemic response reflects the self-interest of the medical science community, building owners and mass transit authorities.

The vaccine strategy assures grants, power, prestige and profits to the medical research community and the drug companies. It enables building owners and mass transit authorities to avoid the cost of providing safe air to customers by hiding behind the medical science community. It results in a government demanding that people wear masks to avoid forcing mass transit authorities and building owners to clean up their virus-contaminated air. For the press the current story has heroes and villains whereas installing air filters and alarms that can stop all air-borne pandemics is boring, low-tech and not newsworthy.
The technology for safe clean air has existed since the 1950s. The cost is small for most of the country-certainly below the cost of clean water and sewer. The savings from just lost time from the flu would likely cover the cost; never mind the cost of this pandemic. Clean filtered air is the only strategy that can prevent future airborne pandemics in a world of very large cities, mass transit and a global air transport network. The problem is political. The resistance to requiring safe air and ultra-cheap air monitors to warn customers when they are breathing other people's air are big-city mayors, mass transit authorities and some building owners. However, if we do not clean up the air in crowded locations, either:

- This virus will mutate around our vaccines and be back or

- $\quad$ Some new natural virus or a virus modified by man will show up to kill off more Americans.

The choice is ours.

NOTE: For this short review article, the following references were use as whole and we encourage that readers of this article refer to these refences for further detailed information.

\section{Acknowledgement}

Additionally, this author is thankful to Professor Charles Forsberg of Massachusetts Institute of Technology (MIT) for his rich and kind contribution.

\section{Bibliography}

1. M Z Bazant and W M Bush. "A guideline to limit indoor airborne transmission of COVID-19". Proceedings of the National Academy of Sciences of the United States of America 118.17 (2021): e2018995118.

2. J Conca. "Engineering Solutions May Work Better on Coronavirus than Social Ones". Forbes, August 11, (2020).

3. L Poon. "What Makes Building Ventilation Good Enough to Withstand a Pandemic?". Bloomberg City lab 11 Jan (2021).

4. R P Siegel. "Shoring up the Front Lines". Mechanical Engineering 143.2 (2021).

5. www.nytimes.com/interactive/2021/02/26/science/

6. S Peacock. "COG-UK Showcase Event, COVID-19 Genomics UK (COG-UK) consortium (COG-UK Showcase Event - YouTube)". 
7. F Lentzos. "How to Protect the World from Ultra Targeted Biological Weapons". Bulletin of the Atomic Scientist (2020).

Volume 5 Issue 7 July 2021

(C) All rights are reserved by Bahman Zohuri. 